\title{
OTHER ARTICLES NOTED (25 Oct 02 to 17 Jan 03)
}

A list of the journals that are reviewed and the criteria for selecting articles from these journals for inclusion in Evidence-Based Nursing are included in each issue. The following articles passed all criteria but were not abstracted because, in the judgment of the editors, their findings were less widely applicable to nursing practice, the topic was of interest to only a select group of nurse specialists, or the topic was recently addressed in another abstract.

\section{Treatment}

Alamo M, Moral R, Pérula de Torres L. Evaluation of a patientcentred approach in generalized musculoskeletal chronic pain/ fibromyalgia patients in primary care. Patient Educ Couns 2002;48:23-31. http://www.ncbi.nlm.nih.gov/entrez/query. fcgi?cmd=Retrieve\&db=PubMed\&list_uids=12220747\&dopt= Abstract

Antman EM. Early administration of intravenous magnesium to high-risk patients with acute myocardial infarction in the Magnesium in Coronaries (MAGIC) Trial: a randomised controlled trial. Lancet 2002;360:1189-96. http://www.ncbi.nlm.nih.gov/ entrez/query.fcgi?cmd=Retrieve\&db=PubMed\&list_uids= $12401244 \&$ dopt=Abstract

Bahl R, Bhandari N, Saksena M, et al. Efficacy of zinc-fortified oral rehydration solution in 6- to 35-month-old children with acute diarrhea.J Pediatr 2002;141:677-82. http://www.ncbi.nlm. nih.gov/entrez/query.fcgi? $\mathrm{cmd}=$ Retrieve $\& \mathrm{db}=\mathrm{PubMed} \&$ list_uids $=12410197 \&$ dopt $=$ Abstract

Ball K, Berch DB, Helmers KF, et al. Effects of cognitive training interventions with older adults: a randomized controlled trial. JAMA 2002;288:2271-81.http://www.ncbi.nlm.nih.gov/entrez/ query.fcgi? $\mathrm{cmd}=$ Retrieve $\& d b=$ PubMed\&list uids $=12425704 \&$ $\mathrm{dopt}=\mathrm{Abstract}$

Baqui AH, Black RE, El Arifeen S, et al. Effect of zinc supplementation started during diarrhoea on morbidity and mortality in Bangladeshi children: community randomised trial. BMJ 2002;325:1059-63. http://www.ncbi.nlm.nih.gov/entrez/ query.fcgi? $\mathrm{cmd}=$ Retrieve $\& d b=$ PubMed\&list_uids $=12424162 \&$ $\mathrm{dopt}=\mathrm{Abstract}$

Barlow J, Parsons J. Group based parent-training programmes for improving emotional and behavioural adjustment in 0-3 year old children. Cochrane Database Syst Rev 2002;(4): CD003680. http://www.ncbi.nlm.nih.gov/entrez/query.fcgi? cmd=Retrieve\&db=PubMed\&list_uids $=12076500 \&$ dopt $=$ Abstract

Barlow J, Wright C, Sheasby J, et al. Self-management approaches for people with chronic conditions: a review. Patient Educ Couns 2002;48:177-87. http://www.ncbi.nlm.nih.gov/ entrez/query.fcgi? $\mathrm{cmd}=$ Retrieve\&db=PubMed\&list_uids= 12401421\&dopt=Abstract

Brandt LJ, Bjorkman D, Fennerty MB, et al. Systematic review on the management of irritable bowel syndrome in North America. Am J Gastroenterol 2002;97:S7-26. http://www.ncbi.nlm.nih.gov/ entrez/query.fcgi? $\mathrm{cmd}=$ Retrieve $\& d b=$ PubMed\&list_uids= $12425586 \&$ dopt $=$ Abstract

Carlo WA, Stark AR, Wright LL, et al. Minimal ventilation to prevent bronchopulmonary dysplasia in extremely-low-birthweight infants. J Pediatr 2002;141:370-4. http://www.ncbi.nlm. nih.gov/entrez/query.fcgi? $\mathrm{cmd}=$ Retrieve\& $\mathrm{db}=$ PubMed\&list_uids $=12219057 \&$ dopt $=$ Abstract

Cole MG, McCusker J, Bellavance F, et al. Systematic detection and multidisciplinary care of delirium in older medical inpatients: a randomized trial. CMAJ 2002;167:753-9. http:// www.ncbi.nlm.nih.gov/entrez/query.fcgi?cmd=Retrieve\&db= PubMed\&list_uids=12389836\&dopt=Abstract

Darlow BA, Graham PJ. Vitamin A supplementation for preventing morbidity and mortality in very low birthweight infants. Cochrane Database Syst Rev 2000;(2):CD000501. http:// www.ncbi.nlm.nih.gov/entrez/query.fcgi?cmd=Retrieve\&db= PubMed\&list_uids=10796372\&dopt=Abstract

de Moor C, Sterner J, Hall M, et al. A pilot study of the effects of expressive writing on psychological and behavioral adjustment in patients enrolled in a Phase II trial of vaccine therapy for metastatic renal cell carcinoma. Health Psychol 2002;21:615-9. http://www.ncbi.nlm.nih.gov/entrez/query.fcgi?cmd=Retrieve $\& d b=$ PubMed\&list_uids=12433015\&dopt=Abstract

Diamond GS, Reis BF, Diamond GM, et al. Attachment-based family therapy for depressed adolescents: a treatment development study. J Am Acad Child Adolesc Psychiatry 2002;41:1190-6. http://www.ncbi.nlm.nih.gov/entrez/query.fcgi?cmd=Retrieve $\& d b=$ PubMed\&list_uids=12364840\&dopt=Abstract

Didjurgeit U, Kruse J, Schmitz N, et al. A time-limited, problemorientated psychotherapeutic intervention in Type 1 diabetic patients with complications: a randomized controlled trial. Diabet Med 2002;19:814-21. http://www.ncbi.nlm.nih.gov/entrez/ query.fcgi? $\mathrm{cmd}=$ Retrieve\&db=PubMed\&list_uids=12358867\& $\mathrm{dopt}=$ Abstract

Dobyns EL, Anas NG, Fortenberry JD, et al. Interactive effects of high-frequency oscillatory ventilation and inhaled nitric oxide in acute hypoxemic respiratory failure in pediatrics. Crit Care Med 2002;30:2425-9. http://www.ncbi.nlm.nih.gov/entrez/ query.fcgi? $\mathrm{cmd}=$ Retrieve $\& \mathrm{db}=$ PubMed\&list_uids $=12441749 \&$ $\mathrm{dopt}=$ Abstract

Dunstan DW, Daly RM, Owen N, et al. High-intensity resistance training improves glycemic control in older patients with type 2 diabetes. Diabetes Care 2002;25:1729-36. http:// www.ncbi.nlm.nih.gov/entrez/query.fcgi?cmd=Retrieve\&db= PubMed\&list_uids=12351469\&dopt=Abstract

Elwy AR, Hart GJ, Hawkes S, et al. Effectiveness of interventions to prevent sexually transmitted infections and human immunodeficiency virus in heterosexual men: a systematic review. Arch Intern Med 2002;162:1818-30. http://www.ncbi.nlm.nih.gov/ entrez/query.fcgi?cmd=Retrieve\&db=PubMed\&list_uids $=$ $12196079 \&$ dopt=Abstract

Fisher B, Anderson S, Bryant J, et al. Twenty-year follow-up of a randomized trial comparing total mastectomy, lumpectomy, and lumpectomy plus irradiation for the treatment of invasive breast cancer. N Engl J Med 2002;347:1233-41. http://www.ncbi.nlm. nih.gov/entrez/query.fcgi?cmd=Retrieve\&db=PubMed\&list_uids $=12393820 \&$ dopt $=$ Abstract

Focht DR 3rd, Spicer C, Fairchok MP. The efficacy of duct tape vs cryotherapy in the treatment of verruca vulgaris (the common wart). Arch Pediatr Adolesc Med 2002;156:971-4. 
http://www.ncbi.nlm.nih.gov/entrez/query.fcgi?cmd=Retrieve $\& \mathrm{db}=$ PubMed\&list_uids $=12361440 \& \mathrm{dopt}=$ Abstract

Furlan AD, Brosseau L, Imamura M, et al. Massage for low-back pain: a systematic review within the framework of the Cochrane Collaboration Back Review Group. Spine 2002;27:1896-910. http://www.ncbi.nlm.nih.gov/entrez/query.fcgi?cmd=Retrieve $\& \mathrm{db}=$ PubMed\&list_uids=12221356\&dopt=Abstract

Furukawa TA, McGuire H, Barbui C. Meta-analysis of effects and side effects of low dosage tricyclic antidepressants in depression: systematic review. BMJ 2002;325:991-5. http://www.ncbi.nlm. nih.gov/entrez/query.fcgi? $c$ cmd=Retrieve $\& d b=P u b M e d \& l i s t \_u i d s$ $=12411354 \&$ dopt $=$ Abstract

Galetta SL, Markowitz C, Lee AG. Immunomodulatory agents for the treatment of relapsing multiple sclerosis: a systematic review. Arch Intern Med 2002;162:2161-9. http:// www.ncbi.nlm.nih.gov/entrez/query.fcgi?cmd=Retrieve\&db= PubMed\&list_uids $=12390057 \&$ dopt $=$ Abstract

Garner S, Fidan D, Frankish R, et al. Celecoxib for rheumatoid arthritis. Cochrane Database Syst Rev 2002;(4):CD003831. http:// www.ncbi.nlm.nih.gov/entrez/query.fcgi? $\mathrm{cmd}=$ Retrieve\&db= PubMed\&list_uids $=12519610 \&$ dopt $=$ Abstract

Gera T, Sachdev HP. Effect of iron supplementation on incidence of infectious illness in children: systematic review. BMJ 2002;325:1142-4. http://www.ncbi.nlm.nih.gov/entrez/query. fcgi?cmd=Retrieve $\& d b=$ PubMed\&list_uids=12433763\&dopt= Abstract

Gill TM, Baker DI, Gottschalk M, et al. A program to prevent functional decline in physically frail, elderly persons who live at home. N Engl J Med 2002;347:1068-74. http://www.ncbi.nlm. nih.gov/entrez/query.fcgi?cmd=Retrieve $\& d b=P u b M e d \& l i s t \_u i d s$ $=12362007 \& \mathrm{dopt}=$ Abstract

Glanz K, Geller AC, Shigaki D, et al. A randomized trial of skin cancer prevention in aquatics settings: the Pool Cool program. Health Psychol 2002;21:579-87. http://www.ncbi.nlm.nih.gov/ entrez/query.fcgi? $\mathrm{cmd}=$ Retrieve $\& d b=$ PubMed\&list_uids $=$ $12433010 \&$ dopt $=$ Abstract

Glasgow RE, Toobert DJ, Hampson SE, Strycker LA. Implementation, generalization and long-term results of the "choosing well" diabetes self-management intervention. Patient Educ Couns 2002;48:115-22. http://www.ncbi.nlm.nih.gov/entrez/query. fcgi? $\mathrm{cmd}=$ Retrieve\&db=PubMed\&list_uids=12401414\&dopt= Abstract

Gotzsche PC, Johansen HK. Nystatin prophylaxis and treatment in severely immunodepressed patients. Cochrane Database Syst Rev 2002;(2):CD002033. http://www.ncbi.nlm.nih.gov/entrez/ query.fcgi? $\mathrm{cmd}=$ Retrieve $\& d b=$ PubMed\&list_uids $=12076436 \&$ $\mathrm{dopt}=\mathrm{Abstract}$

Grant JS, Elliott TR, Weaver M, et al. Telephone intervention with family caregivers of stroke survivors after rehabilitation. Stroke 2002;33:2060-5. http://www.ncbi.nlm.nih.gov/entrez/query. fcgi?cmd=Retrieve\&db=PubMed\&list_uids=12154263\&dopt= Abstract

Haeusler G, Leitich H, van Trotsenburg M, et al. Drug therapy of urinary urge incontinence: a systematic review. Obstet Gynecol 2002;100:1003-16. http://www.ncbi.nlm.nih.gov/entrez/query. fcgi?cmd=Retrieve\&db=PubMed\&list_uids $=12423868 \&$ dopt $=$ Abstract
Harding GK, Zhanel GG, Nicolle LE, et al. Antimicrobial treatment in diabetic women with asymptomatic bacteriuria. $N$ Engl J Med 2002;347:1576-83. http://www.ncbi.nlm.nih.gov/ entrez/query.fcgi?cmd=Retrieve $\& d b=$ PubMed\&list_uids= $12432044 \&$ dopt=Abstract

Hermiz O, Comino E, Marks G, et al. Randomised controlled trial of home based care of patients with chronic obstructive pulmonary disease. BMJ 2002;325:938-40. http://www.ncbi.nlm. nih.gov/entrez/query.fcgi?.cmd=Retrieve\&db=PubMed\&list_uids $=12399344 \& \mathrm{dopt}=$ Abstract

Hurlen M, Abdelnoor M, Smith P, et al. Warfarin, aspirin, or both after myocardial infarction. $N$ Engl J Med 2002;347:969-74. http://www.ncbi.nlm.nih.gov/entrez/query.fcgi?cmd=Retrieve $\& \mathrm{db}=$ PubMed\&list_uids=12324552\&dopt=Abstract

Hurwitz EL, Morgenstern H, Harber P, et al. A randomized trial of medical care with and without physical therapy and chiropractic care with and without physical modalities for patients with low back pain: 6-month follow-up outcomes from the UCLA low back pain study. Spine 2002;27:2193-204. http://www.ncbi.nlm.nih.gov/entrez/query.fcgi?cmd=Retrieve $\& d b=$ PubMed\&list_uids=12394892\&dopt=Abstract

Huusko TM, Karppi P, Avikainen V, et al. Intensive geriatric rehabilitation of hip fracture patients: a randomized, controlled trial. Acta Orthop Scand 2002;73:425-31.http://www.ncbi.nlm.nih. gov/entrez/query.fcgi? $\mathrm{cmd}=$ Retrieve $\& \mathrm{db}=$ PubMed\&list_uids $=$ 12358116\&dopt=Abstract

Kern RS, Liberman RP, Kopelowicz A, et al. Applications of errorless learning for improving work performance in persons with schizophrenia. Am J Psychiatry 2002;159:1921-6. http:// www.ncbi.nlm.nih.gov/entrez/query.fcgi? $\mathrm{cmd}=$ Retrieve\&db= PubMed\&list_uids=12411229\&dopt=Abstract

Keyserling TC, Samuel-Hodge CD, Ammerman AS, et al. A randomized trial of an intervention to improve self-care behaviors of African-American women with type 2 diabetes: impact on physical activity. Diabetes Care 2002;25:1576-83. http://www.ncbi. nlm.nih.gov/entrez/query.fcgi?cmd=Retrieve\&db=PubMed\& list_uids $=12196430 \& d o p t=A b s t r a c t$

Kroeger A, Avila EV, Morison L. Insecticide impregnated curtains to control domestic transmission of cutaneous leishmaniasis in Venezuela: cluster randomised trial. BMJ 2002;325: 810-3. http://www.ncbi.nlm.nih.gov/entrez/query.fcgi?.cmd= Retrieve\&db=PubMed\&list_uids=12376442\&dopt=Abstract

Lafata JE, Baker AM, Divine GW, et al. The use of computerized birthday greeting reminders in the management of diabetes. $J$ Gen Intern Med 2002;17:521-30. http://www.ncbi.nlm.nih.gov/ entrez/query.fcgi? $\mathrm{cmd}=$ Retrieve\&db=PubMed\&list_uids= 12133142\&dopt=Abstract

Laine L, Bombardier C, Hawkey CJ, et al. Stratifying the risk of NSAID-related upper gastrointestinal clinical events: results of a double-blind outcomes study in patients with rheumatoid arthritis. Gastroenterology 2002;123:1006-12. http://www.ncbi. nlm.nih.gov/entrez/query.fcgi?cmd=Retrieve\&db=PubMed\& list_uids=12360461\&dopt=Abstract

Lewis S, Tarrier N, Haddock G, et al. Randomised controlled trial of cognitive-behavioural therapy in early schizophrenia: acutephase outcomes. Br J Psychiatry 2002;43:s91-7. http:// www.ncbi.nlm.nih.gov/entrez/query.fcgi?cmd=Retrieve\&db= PubMed\&list_uids=12271807\&dopt=Abstract 
Mattick RP, Breen C, Kimber J, et al. Methadone maintenance therapy versus no opioid replacement therapy for opioid dependence. Cochrane Database Syst Rev 2002;(2):CD002209. http://www.ncbi.nlm.nih.gov/entrez/query.fcgi?cmd=Retrieve $\& \mathrm{db}=$ PubMed\&list_uids $=12519570 \& d o p t=$ Abstract

McCaul KD, Johnson RJ, Rothman AJ. The effects of framing and action instructions on whether older adults obtain flu shots. Health Psychol 2002;21:624-8. http://www.ncbi.nlm.nih.gov/ entrez/query.fcgi?cmd=Retrieve\&db=PubMed\&list_uids= 12433017\&dopt=Abstract

McCrory DC, Brown CD. Anti-cholinergic bronchodilators versus beta2-sympathomimetic agents for acute exacerbations of chronic obstructive pulmonary disease. Cochrane Database Syst Rev 2002;(4):CD003900. http://www.ncbi.nlm.nih.gov/entrez/ query.fcgi? $\mathrm{cmd}=$ Retrieve $\& d b=$ PubMed\&list_uids $=12519615 \&$ $\mathrm{dopt}=\mathrm{Abstract}$

Meinking TL, Clineschmidt CM, Chen C, et al. An observerblinded study of $1 \%$ permethrin creme rinse with and without adjunctive combing in patients with head lice. $J$ Pediatr 2002;141:665-70. http://www.ncbi.nlm.nih.gov/entrez/query. fcgi? $\mathrm{cmd}=$ Retrieve $\& d b=$ PubMed\&list_uids $=12410195 \&$ dopt $=$ Abstract

Miles JM, Leiter L, Hollander P, et al. Effect of orlistat in overweight and obese patients with type 2 diabetes treated with metformin. Diabetes Care. 2002;25:1123-8. http://www.ncbi.

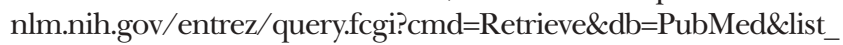
uids $=12087008 \&$ dopt $=$ Abstract

Mørkved S, B $\varnothing$ K, Fjørtoft T. Effect of adding biofeedback to pelvic floor muscle training to treat urodynamic stress incontinence. Obstet Gynecol 2002;100:730-9. http://www.ncbi.nlm.nih. gov/entrez/query.fcgi? cmd=Retrieve $\& \mathrm{db}=$ PubMed\&list_uids= $12383542 \&$ dopt $=$ Abstract

Morris P, Leach A. Antibiotics for persistent nasal discharge (rhinosinusitis) in children. Cochrane Database Syst Rev 2002;(4): CD001094. http://www.ncbi.nlm.nih.gov/entrez/query.fcgi? cmd=Retrieve\&db=PubMed\&list_uids=12519551\&dopt= Abstract

Norris SL, Lau J, Smith SJ, et al. Self-management education for adults with type 2 diabetes: a meta-analysis of the effect on glycemic control. Diabetes Care 2002;25:1159-71. http:// www.ncbi.nlm.nih.gov/entrez/query.fcgi?cmd=Retrieve\&db= PubMed\&list_uids=12087014\&dopt=Abstract

Osman LM, Calder C, Godden DJ, et al. A randomised trial of self-management planning for adult patients admitted to hospital with acute asthma. Thorax 2002;57:869-74. http://www.ncbi. nlm.nih.gov/entrez/query.fcgi? $\mathrm{cmd}=$ Retrieve\&db=PubMed\& list_uids $=12324673 \&$ dopt $=$ Abstract

Piedra PA, Yan L, Kotloff K, et al. Safety of the trivalent, cold-adapted influenza vaccine in preschool-aged children. Pediatrics 2002;110:662-72. http://www.ncbi.nlm.nih.gov/entrez/ query.fcgi?cmd=Retrieve $\& d b=$ PubMed\&list_uids $=12359778 \&$ dopt=Abstract

Pozzilli C, Brunetti M, Amicosante AM, et al. Home based management in multiple sclerosis: results of a randomised controlled trial. J Neurol Neurosurg Psychiatry 2002;73:250-5. http://www.ncbi.nlm.nih.gov/entrez/query.fcgi?cmd= Retrieve\&db=PubMed\&list_uids=12185154\&dopt=Abstract
Quinn J, Cummings S, Callaham M, et al. Suturing versus conservative management of lacerations of the hand: randomised controlled trial. BMJ 2002;325:299-301. http:// www.ncbi.nlm.nih.gov/entrez/query.fcgi? $\mathrm{cmd}=$ Retrieve\&db= PubMed\&list_uids=12169503\&dopt=Abstract

Raine R, Haines A, Sensky T, et al. Systematic review of mental health interventions for patients with common somatic symptoms: can research evidence from secondary care be extrapolated to primary care? BMJ 2002;325:1082-5. http:// www.ncbi.nlm.nih.gov/entrez/query.fcgi? $\mathrm{cmd}=$ Retrieve\&db= PubMed\&list_uids=12424170\&dopt=Abstract

Remington R. Calming music and hand massage with agitated elderly. Nurs Res 2002;51:317-23. http://www.ncbi.nlm.nih.gov/ entrez/query.fcgi?cmd=Retrieve\&db=PubMed\&list_uids= 12352780\&dopt=Abstract

Rostom A, Dube C, Wells G, et al. Prevention of NSAID-induced gastroduodenal ulcers. Cochrane Database Syst Rev 2002;(4): CD002296. http://www.ncbi.nlm.nih.gov/entrez/query.fcgi?cmd $=$ Retrieve $\& \mathrm{db}=$ PubMed\&list_uids=11034748\&dopt=Abstract

Samama CM, Vray M, Barré J, et al. Extended venous thromboembolism prophylaxis after total hip replacement: a comparison of low-molecular-weight heparin with oral anticoagulant. Arch Intern Med 2002;162:2191-6. http://www.ncbi. nlm.nih.gov/entrez/query.fcgi?cmd=Retrieve $\& d b=$ PubMed\& list_uids $=12390061 \&$ dopt $=$ Abstract

Schwedes U, Siebolds M, Mertes G. Meal-related structured selfmonitoring of blood glucose: effect on diabetes control in noninsulin-treated type 2 diabetic patients. Diabetes Care 2002;25: 1928-32. http://www.ncbi.nlm.nih.gov/entrez/query.fcgi?cmd= Retrieve\&db=PubMed\&list_uids=12401734\&dopt=Abstract

Sestini P, Renzoni E, Robinson S, et al. Short-acting beta 2 agonists for stable chronic obstructive pulmonary disease. Cochrane Database Syst Rev 2002;(4):CD001495. http://www.ncbi.nlm. nih.gov/entrez/query.fcgi? $\mathrm{cmd}=$ Retrieve\&db=PubMed\&list_uids $=12519559 \&$ dopt $=$ Abstract

Shepherd J, Blauw GJ, Murphy MB, et al. Pravastatin in elderly individuals at risk of vascular disease (PROSPER): a randomised controlled trial. Lancet 2002;360:1623-30. http://www.ncbi. nlm.nih.gov/entrez/query.fcgi? cmd=Retrieve\&db=PubMed\& list_uids $=12457784 \&$ dopt $=$ Abstract

Shukla VK, Husereau D, Boucher M, et al. Long-acting $\beta_{2}$-agonists for maintenance therapy of stable chronic obstructive pulmonary disease: a systematic review. Ottawa: Canadian Coordinating Office for Health Technology Assessment (CCOHTA), September 2002. Technology report no. 27. http://www.ccohta.ca

Silagy C, Lancaster T, Stead L, et al. Nicotine replacement therapy for smoking cessation. Cochrane Database Syst Rev 2002;(4):CD000146. http://www.ncbi.nlm.nih.gov/entrez/query. fcgi? $\mathrm{cmd}=$ retrieve\&db=pubmed\&list_uids=12519537\&dopt= abstract

Skilnand E, Fossen D, Heiberg E. Acupuncture in the management of pain in labor. Acta Obstet Gynecol Scand 2002;81:943-8. http://www.ncbi.nlm.nih.gov/entrez/query.fcgi ?cmd=Retrieve\&db=PubMed\&list_uids $=12366485 \& \mathrm{dopt}=$ Abstract

Smolen D, Topp R, Singer L. The effect of self-selected music during colonoscopy on anxiety, heart rate, and blood pressure. Appl Nurs Res 2002;15:126-36. http://www.ncbi.nlm.nih.gov/ 
entrez/query.fcgi? $\mathrm{cmd}=$ Retrieve\&db=PubMed\&list_uids= $12173164 \&$ dopt $=$ Abstract

Swanson JM, Gupta S, Williams L, et al. Efficacy of a new pattern of delivery of methylphenidate for the treatment of ADHD: effects on activity level in the classroom and on the playground. J Am Acad Child Adolesc Psychiatry 2002;41:1306-14. http:// www.ncbi.nlm.nih.gov/entrez/query.fcgi?cmd=Retrieve\&db= PubMed\&list_uids=12410072\&dopt=Abstract

Szilagyi P, Vann J, Bordley C, et al. Interventions aimed at improving immunization rates. Cochrane Database Syst Rev 2002;(4):CD003941. http://www.ncbi.nlm.nih.gov/entrez/query. fcgi? $c$ dendrieve \&db=PubMed\&list_uids=12519624\&dopt= Abstract

Thomas R. School-based programmes for preventing smoking. Cochrane Database Syst Rev 2002;(4):CD001293. http://www. ncbi.nlm.nih.gov/entrez/query.fcgi? $\mathrm{cmd}=$ Retrieve $\& \mathrm{db}=\mathrm{PubMed}$ \&list_uids=12519553\&dopt=Abstract

van den Broek N, Kulier R, Gülmezoglu AM, et al. Vitamin A supplementation during pregnancy. Cochrane Database Syst Rev 2002;(4):CD001996. http://www.ncbi.nlm.nih.gov/entrez/query. fcgi? cmd=Retrieve\&db=PubMed\&list_uids=12519564\&dopt= Abstract

Veronesi U, Cascinelli N, Mariani L, et al. Twenty-year follow-up of a randomized study comparing breast-conserving surgery with radical mastectomy for early breast cancer. $N$ Engl J Med 2002;347:1227-32. http://www.ncbi.nlm.nih.gov/entrez/query. fcgi? cmd=Retrieve\&db=PubMed\&list_uids=12393819\&dopt= Abstract

von Hertzen H, Piaggio G, Ding J, et al. Low dose mifepristone and two regimens of levonorgestrel for emergency contraception: a WHO multicentre randomised trial. Lancet 2002;360:1803-10. http://www.ncbi.nlm.nih.gov/entrez/query. fcgi?cmd=Retrieve\&db=PubMed\&list_uids=12480356\&dopt= Abstract

Washington DL, Stevens CD, Shekelle PG, et al. Next-day care for emergency department users with nonacute conditions. A randomized, controlled trial. Ann Intern Med 2002;137:707-14. http://www.ncbi.nlm.nih.gov/entrez/query.fcgi?cmd=Retrieve $\& \mathrm{db}=$ PubMed\&list_uids $=12416944 \& d o p t=$ Abstract

Weingarten SR, Henning JM, Badamgarav E, et al. Interventions used in disease management programmes for patients with chronic illness-which ones work? Meta-analysis of published reports. BMJ 2002;325:925-8. http://www.ncbi.nlm.nih.gov/ entrez/query.fcgi?cmd=Retrieve\&db=PubMed\&list_uids= 12399340\&dopt=Abstract

Weisman SM, Graham DY. Evaluation of the benefits and risks of low-dose aspirin in the secondary prevention of cardiovascular and cerebrovascular events. Arch Intern Med 2002;162:2197202. http://www.ncbi.nlm.nih.gov/entrez/query.fcgi?cmd= Retrieve\&db=PubMed\&list_uids=12390062\&dopt=Abstract

Wilkinson D, Ramjee G, Tholandi M, et al. Nonoxynol-9 for preventing vaginal acquisition of sexually transmitted infections by women from men. Cochrane Database Syst Rev 2002;(4): CD003936. http://www.ncbi.nlm.nih.gov/entrez/query.fcgi?cmd $=$ Retrieve\&db=PubMed\&list_uids=12519623\&dopt=Abstract

Wyatt KM, Dimmock PW, O'Brien PM. Selective serotonin reuptake inhibitors for premenstrual syndrome. Cochrane Database Syst Rev 2002;(4):CD001396. http:/ /www.ncbi.nlm.nih.gov/ entrez/query.fcgi? $\mathrm{cmd}=$ Retrieve\&db=PubMed\&list_uids= $12519554 \&$ dopt=Abstract

Younes RN, Gross JL, Aguiar S, et al. When to remove a chest tube? A randomized study with subsequent prospective consecutive validation.J Am Coll Surg 2002;195:658-62. http:// www.ncbi.nlm.nih.gov/entrez/query.fcgi?cmd=Retrieve\&db= PubMed\&list_uids=12437253\&dopt=Abstract

Zhu SH, Anderson CM, Tedeschi GJ, et al. Evidence of real-world effectiveness of a telephone quitline for smokers. $N \mathrm{Engl} \mathrm{J} \mathrm{Med}$ 2002;347:1087-93. http://www.ncbi.nlm.nih.gov/entrez/query. fcgi? $\mathrm{cmd}=$ Retrieve $\& d b=$ PubMed\&list_uids $=12362011 \& d o p t=$ Abstract

Zygmunt A, Olfson M, Boyer CA, et al. Interventions to improve medication adherence in schizophrenia. Am J Psychiatry 2002;159:1653-64. http://www.ncbi.nlm.nih.gov/entrez/query. fcgi? $c m d=$ Retrieve $\& d b=$ PubMed\&list_uids $=12359668 \& d o p t=$ Abstract

\section{Assessment (screening and diagnosis)}

Kulasingam SL, Hughes JP, Kiviat NB, et al. Evaluation of human papillomavirus testing in primary screening for cervical abnormalities: comparison of sensitivity, specificity, and frequency of referral. JAMA 2002;288:1749-57. http://www.ncbi.nlm.nih. gov/entrez/query.fcgi?cmd=Retrieve $\& \mathrm{db}=$ PubMed\&list_uids= $12365959 \& d o p t=$ Abstract

Powlishta KK, Von Dras DD, Stanford A, et al. The clock drawing test is a poor screen for very mild dementia. Neurology 2002;59:898-903. http://www.ncbi.nlm.nih.gov/entrez/query. fcgi? cmd=Retrieve $\& d b=$ PubMed\&list_uids $=12297574 \& d o p t=$ Abstract

\section{Prognosis}

Boiko A, Vorobeychik G, Paty D, et al. Early onset multiple sclerosis: a longitudinal study. Neurology 2002;59:1006-10. http:// www.ncbi.nlm.nih.gov/entrez/query.fcgi? $\mathrm{cmd}=$ Retrieve\&db= PubMed\&list_uids=12370453\&dopt=Abstract

Cicognani A, Alessandroni R, Pasini A, et al. Low birth weight for gestational age and subsequent male gonadal function.J Pediatr 2002;141:376-9. http://www.ncbi.nlm.nih.gov/entrez/query. fcgi? cmd=Retrieve\&db=PubMed\&list_uids=12219058\&dopt= Abstract

Joyce E, Hutton S, Mutsatsa S, et al. Executive dysfunction in first-episode schizophrenia and relationship to duration of untreated psychosis: the West London Study. Br J Psychiatry 2002;43:s38-44. http://www.ncbi.nlm.nih.gov/entrez/query. fcgi? $c$ dd $=$ Retrieve $\& d b=$ PubMed\&list_uids $=12271799 \& d o p t=$ Abstract

Linné Y, Barkeling B, Rössner S. Natural course of gestational diabetes mellitus: long term follow up of women in the SPAWN study. BJOG 2002;109:1227-31. http://www.ncbi.nlm.nih.gov/ entrez/query.fcgi?cmd=Retrieve\&db=PubMed\&list_uids= 12452459\&dopt=Abstract

Starc TJ, Lipshultz SE, Easley KA, et al. Incidence of cardiac abnormalities in children with human immunodeficiency virus infection: the prospective P2C2 HIV study. J Pediatr 2002;141:327-34. http://www.ncbi.nlm.nih.gov/entrez/query. fcgi? cmd=Retrieve $\& d b=$ PubMed\&list_uids $=12219051 \& d o p t=$ Abstract 
Tsai PS. White coat hypertension: understanding the concept and examining the significance. J Clin Nurs 2002;11:715-22. http://www.ncbi.nlm.nih.gov/entrez/query.fcgi?cmd=Retrieve $\& d b=$ PubMed\&list_uids $=12427175 \& d o p t=$ Abstract

Vidaillet H, Granada JF, Chyou PH, et al. A population-based study of mortality among patients with atrial fibrillation or flutter. Am J Med 2002;113:365-70. http://www.ncbi.nlm.nih.gov/ entrez/query.fcgi? $\mathrm{cmd}=$ Retrieve $\& \mathrm{db}=\mathrm{PubMed} \&$ list uids= 12401530\&dopt=Abstract

\section{Causation}

Hoek G, Brunekreef B, Goldbohm S, et al. Association between mortality and indicators of traffic-related air pollution in the Netherlands: a cohort study. Lancet 2002;360:1203-9. http:// www.ncbi.nlm.nih.gov/entrez/query.fcgi? $\mathrm{cmd}=$ Retrieve $\& \mathrm{db}=$ PubMed\&list_uids=12401246\&dopt=Abstract

Kuh D, Hardy R, Langenberg C, et al. Mortality in adults aged 26-54 years related to socioeconomic conditions in childhood and adulthood: post war birth cohort study. BMJ 2002;325: 1076-80. http://www.ncbi.nlm.nih.gov/entrez/query.fcgi?cmd= Retrieve\&db=PubMed\&list_uids=12424168\&dopt=Abstract

Malyutina S, Bobak M, Kurilovitch S, et al. Relation between heavy and binge drinking and all-cause cardiovascular mortality in Novosibirsk, Russia: a prospective cohort study. Lancet Published online 2002 Oct 1. http://image.thelancet.com/ extras/01art11207web.pdf

Manor O, Eisenbach Z. Mortality after spousal loss: are there socio-demographic differences? Soc Sci Med 2003;56:405-13. http://www.ncbi.nlm.nih.gov/entrez/query.fcgi?cmd=Retrieve $\& d b=$ PubMed\&list_uids $=12473324 \& d o p t=$ Abstract

Mondestin MA, Ananth CV, Smulian JC, et al. Birth weight and fetal death in the United States: the effect of maternal diabetes during pregnancy. Am J Obstet Gynecol 2002;187:922-6. http:// www.ncbi.nlm.nih.gov/entrez/query.fcgi?cmd=Retrieve\&db= PubMed\&list_uids $=12388978 \&$ \&opt $=$ Abstract

Qin P, Agerbo E, Bo Mortensen P. Suicide risk in relation to family history of completed suicide and psychiatric disorders: a nested case-control study based on longitudinal registers. Lancet 2002;360:1126-30. http://www.ncbi.nlm.nih.gov/entrez/query. fcgi? $\mathrm{cmd}=$ Retrieve\&db=PubMed\&list_uids $=12387960 \&$ dopt $=$ Abstract

Orr ST, James SA, Blackmore Prince C. Maternal prenatal depressive symptoms and spontaneous preterm births among African-American women in Baltimore, Maryland. Am J Epidemiol 2002;156:797-802. http://www.ncbi.nlm.nih.gov/ entrez/query.fcgi?cmd=Retrieve $\& d b=$ PubMed\&list_uids= 12396996\&dopt=Abstract

Stewart S, Hart CL, Hole DJ, et al. A population-based study of the long-term risks associated with atrial fibrillation: 20-year follow-up of the Renfrew/Paisley study. Am J Med 2002;113: 359-64. http://www.ncbi.nlm.nih.gov/entrez/query.fcgi?cmd= Retrieve\&db=PubMed\&list_uids=12401529\&dopt=Abstract

\section{Quality improvement}

Burns T, White I, Byford S, et al. Exposure to case management: relationships to patient characteristics and outcome. Report from the UK700 trial. Br J Psychiatry 2002;181:236-41.
http://www.ncbi.nlm.nih.gov/entrez/query.fcgi?cmd=Retrieve $\& d b=$ PubMed\&list_uids $=12204929 \&$ dopt=Abstract

Eccles M, McColl E, Steen N, et al. Effect of computerised evidence based guidelines on management of asthma and angina in adults in primary care: cluster randomised controlled trial. BMJ 2002;325:941-4. http://www.ncbi.nlm.nih.gov/entrez/ query.fcgi? $\mathrm{cmd}=$ Retrieve $\& \mathrm{db}=$ PubMed\&list_uids $=12399345 \&$ dopt=Abstract

Harer Jr WB, Valenzuela G, Lebo D. Lubrication of the vaginal introitus and speculum does not affect Papanicolaou smears. Obstet Gynecol 2002;100:887-8. http://www.ncbi.nlm.nih.gov/ entrez/query.fcgi?cmd=Retrieve\&db=PubMed\&list_uids= $12423847 \&$ dopt=Abstract

\section{Economics}

Glasziou PP, Eckermann SD, Mulray SE, et al. Cholesterollowering therapy with pravastatin in patients with average cholesterol levels and established ischaemic heart disease: is it costeffective? Med J Aust 2002;177:428-34. http://www.ncbi.nlm.nih. gov/entrez/query.fcgi?cmd=Retrieve\&db=PubMed\&list_uids= $12381252 \&$ dopt $=$ Abstract

Jacobs P, Finer NN, Fassbender K, et al. Cost-effectiveness of inhaled nitric oxide in near-term and term infants with respiratory failure: eighteen- to 24-month follow-up for Canadian patients. Crit Care Med 2002;30:2330-4. http://www.ncbi.nlm.nih. gov/entrez/query.fcgi? $\mathrm{cmd}=$ Retrieve $\& \mathrm{db}=$ PubMed\&list_uids= $12394963 \&$ dopt=Abstract

Khan K, Muennig P, Behta M, et al. Global drug-resistance patterns and the management of latent tuberculosis infection in immigrants to the United States. N Engl J Med 2002;347:1850-9. http://www.ncbi.nlm.nih.gov/entrez/query.fcgi?cmd=Retrieve\& $\mathrm{db}=$ PubMed\&list_uids $=12466510$ \&dopt=Abstract

Kumar K, Hunter G, Demeria DD. Treatment of chronic pain by using intrathecal drug therapy compared with conventional pain therapies: a cost-effectiveness analysis. J Neurosurg 2002;97:803-10. http://www.ncbi.nlm.nih.gov/entrez/query. fcgi? cmd=Retrieve\&db=PubMed\&list_uids=12405366\&dopt= Abstract

Multiple Aneurysm Screening Study Group. Multicentre aneurysm screening study (MASS): cost effectiveness analysis of screening for abdominal aortic aneurysms based on four year results from randomised controlled trial. BMJ 2002;325:1135-41. http://www.ncbi.nlm.nih.gov/entrez/query.fcgi?.cmd=Retrieve $\& d b=$ PubMed\&list_uids=12433761\&dopt=Abstract

Schermer TR, Thoonen BP, Van Den Boom G, et al. Randomized controlled economic evaluation of asthma selfmanagement in primary health care. Am J Respir Crit Care Med 2002;166:1062-1072. http://www.ncbi.nlm.nih.gov/entrez/ query.fcgi? cmd=Retrieve\&db=PubMed\&list_uids=12379549\& dopt=Abstract

\section{Clinical prediction guide}

Haugeberg G, Ørstavik RE, Uhlig T, et al. Clinical decision rules in rheumatoid arthritis: do they identify patients at high risk for osteoporosis? Testing clinical criteria in a population based cohort of patients with rheumatoid arthritis recruited from the Oslo Rheumatoid Arthritis Register. Ann Rheum Dis 2002;61: 1085-9. http://www.ncbi.nlm.nih.gov/entrez/query.fcgi?cmd= Retrieve\&db=PubMed\&list_uids $=12429540 \&$ dopt=Abstract 
Paterniti S, Verdier-Taillefer MH, Dufouil C, et al. Depressive symptoms and cognitive decline in elderly people. Longitudinal study. Br J Psychiatry 2002;181:406-10. http://www.ncbi.nlm.nih. gov/entrez/query.fcgi? $\mathrm{cmd}=$ Retrieve $\& \mathrm{db}=$ PubMed\&list_uids $=$ 12411266\&dopt=Abstract

Samaha FF, Kimmel SE, Kizer JR, et al. Usefulness of the TIMI risk score in predicting both short- and long-term outcomes in the Veterans Affairs Non-Q-Wave Myocardial Infarction Strategies In-Hospital (VANQWISH) Trial. Am J Cardiol 2002;90:922-6. http://www.ncbi.nlm.nih.gov/entrez/query.fcgi? $\mathrm{cmd}=$ Retrieve $\& \mathrm{db}=$ PubMed\&list_uids $=12398955 \& \mathrm{dopt}=\mathrm{Abstract}$

Scirica BM, Cannon CP, McCabe CH, et al. Prognosis in the thrombolysis in myocardial ischemia III registry according to the Braunwald unstable angina pectoris classification. Am J Cardiol 2002;90:821-6. http://www.ncbi.nlm.nih.gov/entrez/ query.fcgi? $\mathrm{cmd}=$ Retrieve $\& d b=$ PubMed\&list_uids $=12372567 \&$ dopt=Abstract

\section{Qualitative}

Ahmad WI, Atkin K, Jones L. Being deaf and being other things: young Asian people negotiating identities. Soc Sci Med 2002;55:1757-69. http://www.ncbi.nlm.nih.gov/entrez/query. fcgi? $\mathrm{cmd}=$ Retrieve\&db=PubMed\&list_uids $=12383460 \&$ dopt $=$ Abstract

Barnes G, Baxter J, Litva A, et al. The social and psychological impact of the chemical contamination incident in Weston Village, UK: a qualitative analysis. Soc Sci Med 2002;55:2227-41. http://www.ncbi.nlm.nih.gov/entrez/query.fcgi?cmd=Retrieve $\& \mathrm{db}=$ PubMed\&list_uids=12409136\&dopt=Abstract

Carter B, Lambrenos K, Thursfield J. A pain workshop: an approach to eliciting the views of young people with chronic pain.J Clin Nurs 2002;11:753-62. http://www.ncbi.nlm.nih.gov/ entrez/query.fcgi?cmd=Retrieve $\& d b=$ PubMed\&list_uids $=$ $12427180 \&$ dopt $=$ Abstract

Carter PA. Caregivers' descriptions of sleep changes and depressive symptoms. Oncol Nurs Forum 2002;29:1277-83. http://www.ncbi.nlm.nih.gov/entrez/query.fcgi?cmd=Retrieve $\& \mathrm{db}=$ PubMed\&list_uids=12370697\&dopt=Abstract

Chan SW, Levy V, Chung TK, et al. A qualitative study of the experiences of a group of Hong Kong Chinese women diagnosed with postnatal depression. J Adv Nurs 2002;39:571-9. http://www.ncbi.nlm.nih.gov/entrez/query.fcgi?cmd=Retrieve $\& \mathrm{db}=$ PubMed\&list_uids $=12207755 \& d o p t=$ Abstract

Chapple A, Ziebland S, Shepperd S, et al. Why men with prostate cancer want wider access to prostate specific antigen testing: qualitative study. BMJ 2002;325:737-9. http://www.ncbi.nlm.nih. gov/entrez/query.fcgi? $\mathrm{cmd}=$ Retrieve\&db=PubMed\&list_uids= $12364299 \&$ dopt=Abstract

Cheung N. Choice and control as experienced by Chinese and Scottish childbearing women in Scotland. Midwifery 2002;18: 200-13. http://www.ncbi.nlm.nih.gov/entrez/query.fcgi? $\mathrm{cmd}=$ Retrieve\&db=PubMed\&list_uids $=12381424 \&$ dopt=Abstract

Clark AM, Barbour RS, McIntyre PD. Preparing for change in the secondary prevention of coronary heart disease: a qualitative evaluation of cardiac rehabilitation within a region of Scotland. J Adv Nurs 2002;39:589-98. http://www.ncbi.nlm.nih. gov/entrez/query.fcgi?cmd=Retrieve\&db=PubMed\&list_uids= 12207757 \&dopt=Abstract
Davis RE. "The strongest women": exploration of the inner resources of abused women. Qual Health Res 2002;12:1248-63. http://www.ncbi.nlm.nih.gov/entrez/query.fcgi?.cmd=Retrieve $\& \mathrm{db}=$ PubMed\&list_uids=12448670\&dopt=Abstract

Deschepper R, Vander Stichele RH, Haaijer-Ruskamp FM. Cross-cultural differences in lay attitudes and utilisation of antibiotics in a Belgian and a Dutch city. Patient Educ Couns 2002; 48:161-9. http://www.ncbi.nlm.nih.gov/entrez/query.fcgi?cmd= Retrieve\&db=PubMed\&list_uids $=12401419 \&$ dopt $=$ Abstract

Draper J. 'It's the first scientific evidence': men's experience of pregnancy confirmation. J Adv Nurs 2002;39:563-70. http:// www.ncbi.nlm.nih.gov/entrez/query.fcgi?cmd=Retrieve\&db= PubMed\&list_uids $=12207754 \&$ dopt=Abstract

Egerod I. Uncertain terms of sedation in ICU. How nurses and physicians manage and describe sedation for mechanically ventilated patients. J Clin Nurs 2002;1 1:831-40. http://www.ncbi. nlm.nih.gov/entrez/query.fcgi?.cmd=Retrieve\&db=PubMed\& list_uids $=12427190 \&$ dopt $=$ Abstract

Eijkelberg IM, Mur-Veeman IM, Spreeuwenberg C, et al. Patient focus groups about nurse-led shared care for the chronically ill. Patient Educ Couns 2002;47:329-36. http://www.ncbi.nlm.nih. gov/entrez/query.fcgi? $c$ de $=$ Retrieve\& $\mathrm{db}=$ PubMed\&list_uids $=$ $12135824 \&$ dopt=Abstract

Evans JA. Cautious caregivers: gender stereotypes and the sexualization of men nurses' touch. J Adv Nurs 2002;40:441-8. http://www.ncbi.nlm.nih.gov/entrez/query.fcgi?cmd=Retrieve $\& \mathrm{db}=$ PubMed\&list_uids $=12421403 \&$ dopt $=$ Abstract

Fish J, Wilkinson S. Understanding lesbians' healthcare behaviour: the case of breast self-examination. Soc Sci Med 2003;56: 235-45. http://www.ncbi.nlm.nih.gov/entrez/query.fcgi? cmd= Retrieve\&db=PubMed\&list_uids $=12473310 \&$ dopt=Abstract

Fredriksson L, Lindström UA. Caring conversationspsychiatric patients' narratives about suffering. J Adv Nurs 2002; 40:396-404. http://www.ncbi.nlm.nih.gov/entrez/query.fcgi? cmd=Retrieve\&db=PubMed\&list_uids $=12421398 \& d o p t=$ Abstract

Gabe J, Bury M, Ramsay R. Living with asthma: the experiences of young people at home and at school. Soc Sci Med 2002; 55:1619-33. http://www.ncbi.nlm.nih.gov/entrez/query.fcgi? cmd=Retrieve $\& d b=$ PubMed\&list_uids=12297247\&dopt=Abstract

Hörnsten A, Norberg A, Lundman B. Psychosocial maturity among people with diabetes mellitus. J Clin Nurs 2002;11:777-84. http://www.ncbi.nlm.nih.gov/entrez/query.fcgi? cmd=Retrieve $\& \mathrm{db}=$ PubMed\&list_uids=12427183\&dopt=Abstract

Hsu MT, Tseng YF, Kuo LL. Transforming loss: Taiwanese women's adaptation to stillbirth. $J$ Adv Nurs 2002;40:387-95. http://www.ncbi.nlm.nih.gov/entrez/query.fcgi? cmd=Retrieve $\& \mathrm{db}=$ PubMed\&list_uids=12421397\&dopt=Abstract

Hussein S, Partridge M. Perceptions of asthma in South Asians and their views on educational materials and self-management plans: a qualitative study. Patient Educ Couns 2002;48:189-94. http://www.ncbi.nlm.nih.gov/entrez/query.fcgi?cmd=Retrieve $\& \mathrm{db}=$ PubMed\&list_uids=12401422\&dopt=Abstract

Kerr EE, Fothergill-Bourbonnais F. The recovery mosaic: older women's lived experiences after a myocardial infarction. Heart Lung 2002;31:355-67. http://www.ncbi.nlm.nih.gov/entrez/ query.fcgi?.cmd=Retrieve\&db=PubMed\&list_uids $=12487014 \&$ $\mathrm{dopt}=$ Abstract 
Lacey MD. The experience of using decisional support aids by patients with breast cancer. Oncol Nurs Forum 2002;29:1491-7. http://www.ncbi.nlm.nih.gov/entrez/query.fcgi?cmd=Retrieve $\& d b=$ PubMed\&list_uids $=12432419 \& d o p t=$ Abstract

Landmark BT, Wahl A. Living with newly diagnosed breast cancer: a qualitative study of 10 women with newly diagnosed breast cancer. J Adv Nurs 2002;40:112-21. http://www.ncbi.nlm.nih. gov/entrez/query.fcgi?cmd=Retrieve\&db=PubMed\&list_uids= $12230536 \& d o p t=$ Abstract

Lendahls L, Ohman L, Liljestrand J, et al. Women's experiences of smoking during and after pregnancy as ascertained two to three years after birth. Midwifery 2002;18:214-22. http:// www.ncbi.nlm.nih.gov/entrez/query.fcgi? $\mathrm{cmd}=$ Retrieve\&db= PubMed\&list_uids=12381425\&dopt=Abstract

Main J. Management of relatives of patients who are dying. J Clin Nurs 2002;11:794-801. http://www.ncbi.nlm.nih.gov/entrez/ query.fcgi? $\mathrm{cmd}=$ Retrieve $\& d b=$ PubMed\&list_uids $=12427185 \&$ $\mathrm{dopt}=\mathrm{Abstract}$

Matthews AK, Peterman AH, Delaney P, et al. A qualitative exploration of the experiences of lesbian and heterosexual patients with breast cancer. Oncol Nurs Forum 2002;29:1455-62. http://www.ncbi.nlm.nih.gov/entrez/query.fcgi?cmd=Retrieve $\& \mathrm{db}=$ PubMed\&list_uids=12432416\&dopt=Abstract

McKeever P, O’Neill S, Miller KL. Managing space and marking time: mothering severely ill infants in hospital isolation. Qual Health Res 2002;12:1020-32. http://www.ncbi.nlm.nih.gov/entrez/ query.fcgi? $\mathrm{cmd}=$ Retrieve $\&$ db=PubMed\&list_uids $=12365586 \&$ dopt=Abstract

Messecar DC, Archbold PG, Stewart BJ, et al. Home environmental modification strategies used by caregivers of elders. Res Nurs Health 2002;25:357-70. http://www.ncbi.nlm.nih.gov/entrez/ query.fcgi? $c$ cmd=Retrieve\&db=PubMed\&list_uids=12221690\&dopt $=$ Abstract

Murray SA, Boyd K, Kendall M, et al. Dying of lung cancer or cardiac failure: prospective qualitative interview study of patients and their carers in the community. BMJ 2002;325:929-32. http:// www.ncbi.nlm.nih.gov/entrez/query.fcgi?cmd=Retrieve\&db= PubMed\&list_uids=12399341\&dopt=Abstract

O'Brien B, Evans M, White-McDonald E. Isolation from "being alive": coping with severe nausea and vomiting of pregnancy. Nurs Res 2002;51:302-8. http://www.ncbi.nlm.nih.gov/entrez/ query.fcgi? $\mathrm{cmd}=$ Retrieve $\& d b=$ PubMed\&list_uids $=12352778 \&$ $\mathrm{dop} \mathrm{t}=$ Abstract

Paulson M, Norberg A, Danielson E. Men living with fibromyalgia-type pain: experiences as patients in the Swedish health care system. J Adv Nurs 2002;40:87-95. http:// www.ncbi.nlm.nih.gov/entrez/query.fcgi? $\mathrm{cmd}=$ Retrieve $\& \mathrm{db}=$ PubMed\&list_uids=12230533\&dopt=Abstract

Perry J. Wives giving care to husbands with Alzheimer's disease: a process of interpretive caring. Res Nurs Health 2002;25:307-16.
http://www.ncbi.nlm.nih.gov/entrez/query.fcgi?cmd=Retrieve $\& \mathrm{db}=$ PubMed\&list_uids=12124724\&dopt=Abstract

Piggot J, Paterson J, Hocking C. Participation in home therapy programs for children with cerebral palsy: a compelling challenge. Qual Health Res 2002;12:1112-29. http:// www.ncbi.nlm.nih.gov/entrez/query.fcgi?cmd=Retrieve\&db= PubMed\&list_uids=12365591\&dopt=Abstract

Plano Clark VL, Miller DL, Creswell JW, et al. In conversation: high school students talk to students about tobacco use and prevention strategies. Qual Health Res 2002;12:1264-83. http:// www.ncbi.nlm.nih.gov/entrez/query.fcgi? $c m d=$ Retrieve\&db= PubMed\&list_uids=12448671\&dopt=Abstract

Pollock K, Grime J. Patients' perceptions of entitlement to time in general practice consultations for depression: qualitative study. BMJ 2002;325:687-90. http://www.ncbi.nlm.nih.gov/entrez/ query.fcgi? $\mathrm{cmd}=$ Retrieve\&db=PubMed\&list_uids=12351362\& $\mathrm{dopt}=$ Abstract

Richardson J. Health promotion in palliative care: the patients' perception of therapeutic interaction with the palliative nurse in the primary care setting. J Adv Nurs 2002;40:432-40. http:// www.ncbi.nlm.nih.gov/entrez/query.fcgi?cmd=Retrieve\&db= PubMed\&list_uids=12421402\&dopt=Abstract

Ross L, Johansen C. Psychosocial home visits in cancer treatment: a qualitative study on the content of home visits. Cancer Nurs 2002;25:350-7. http://www.ncbi.nlm.nih.gov/entrez/ query.fcgi? $\mathrm{cmd}=$ Retrieve $\& \mathrm{db}=$ PubMed\&list_uids=12394562\& dopt=Abstract

Simpson EM, Moll EK, Kassam-Adams N, et al. Barriers to booster seat use and strategies to increase their use. Pediatrics 2002;110:729-36. http://www.ncbi.nlm.nih.gov/entrez/query. fcgi?cmd=Retrieve $\& d b=$ PubMed\&list_uids $=12359786 \& d o p t=$ Abstract

Tashiro CJ. Considering the significance of ancestry through the prism of mixed-race identity. ANS Adv Nurs Sci 2002;25:1-21. http://www.ncbi.nlm.nih.gov/entrez/query.fcgi?cmd=Retrieve $\& d b=$ PubMed\&list_uids=12484638\&dopt=Abstract

Tod AM, Lacey EA, McNeill F. 'I'm still waiting...': barriers to accessing cardiac rehabilitation services. $J$ Adv Nurs 2002;40: 421-31. http://www.ncbi.nlm.nih.gov/entrez/query.fcgi? cmd= Retrieve\&db=PubMed\&list_uids=12421401\&dopt=Abstract

Twinn S, Shiu AT, Holroyd E. Women's knowledge about cervical cancer and cervical screening practice: a pilot study of Hong Kong Chinese women. Cancer Nurs 2002;25:377-84. http:// www.ncbi.nlm.nih.gov/entrez/query.fcgi?cmd=Retrieve\&db= PubMed\&list_uids=12394564\&dopt=Abstract

Williams C, Alderson P, Farsides B. What constitutes 'balanced information in the practitioners' portrayals of Down's syndrome? Midwifery 2002;18:230-7. http://www.ncbi.nlm.nih.gov/entrez/ query.fcgi? $\mathrm{cmd}=$ Retrieve $\& \mathrm{db}=$ PubMed\&list_uids $=12381427 \&$ dopt=Abstract 